\title{
Strain-Induced Shifts of the Zone-Center Phonons of III-Nitrides
}

\author{
H. W. Leite Alves*, J. L. A. Alves*, A. M. Santos ${ }^{\dagger}$, L. M. R. Scolfaro ${ }^{\dagger}$, and J. R. Leite ${ }^{\dagger}$ \\ * Departamento de Ciências Naturais, Universidade Federal de São João del Rei \\ Caixa Postal 110, 36.300-000, São João del Rei, MG, Brazil \\ † Instituto de Física, Universidade de São Paulo \\ Caixa Postal 66318, 05315-970, São Paulo, SP, Brazil
}

Received on 31 March, 2003

\begin{abstract}
In this work, by using the density-functional theory within the local density approximation, plane wave expansions and the pseudopotential method, we present our preliminary results for the strain-induced shifts of the zone center phonon modes and of the effective charges on the III-Nitrides. Our results are in good agreement with the experiment as well as with other calculations, whenever a comparison is possible.
\end{abstract}

\section{Introduction}

The group III-Nitrides (AlN, GaN, InN) and the corresponding alloys have attracted great interest due to their successful applications in the electronic and optoelectronic device technology [1]. However, their growth in the zincblende structure has been a hard task to the experimentalists, since the most stable structure for these compounds is the wurtzite one. Moreover, by using MBE or MOCVD growth techniques, the lattice matching between the III-Nitrides and the most used substrates (such as $\mathrm{Al}_{2} \mathrm{O}_{3}, \mathrm{SiC}$ or $\mathrm{GaAs}$ ) is around $5 \%$, leading to strained samples with large contents of structural induced defects. So, in order to avoid such defects, Nitride buffer-layers are introduced but the resulting sample presents hexagonal modifications instead of the desired cubic one.

From the theoretical point of view, while the structural and electronic properties of III-Nitrides were extensively studied in the last years, the amount of knowledge on their dynamical properties are rather scarce: only the description of the phonon dispersion of the unstrained cubic modification was done [2]. In order to supply the missing information on the vibrational properties of strained III-Nitrides, we have calculated $a b$ initio the strain-induced shifts of the zone center phonon modes and the effective charges for the IIINitrides, by using the density-functional theory within the local density approximation (LDA), plane wave expansions and the pseudopotential method (ABINIT code) [3]. We have used the Troullier-Martins pseudopotentials, following the recipe developed by Stampfl and Van de Walle [4] for the III-Nitrides, which includes the d-electrons as valence states for $\mathrm{GaN}$ and $\mathrm{InN}$ (using the fhi98PP code [5]). The phonon modes and their dispersion curves were obtained using the adiabatic perturbation theory [6]. Details about the converged bulk equilibrium properties of $\mathrm{AlN}$, GaN and $\mathrm{InN}$ and their unstrained phonon dispersions were described in our previous work [7].

\section{Hydrostatic strains}

In Figs. 1, 2 and 3, we show the stress dependence of the frequencies of the zone-center phonons of $\mathrm{AlN}, \mathrm{GaN}$ and InN, respectively, under a hydrostatic compressive force. It is interesting to note that, as the hydrostatic strains preserve the crystal symmetry, there is no induced splittings of the TO modes. So, the obtained stress dependence can be expressed, by a linear fit, as (in $\mathrm{cm}^{-1} / \mathrm{GPa}$ units):

i) $\Delta \omega_{L O}=(4.32 \pm 0.06) . \varepsilon$ and $\Delta \omega_{T O}=(4.29 \pm 0.06) . \varepsilon$ for AlN;

ii) $\Delta \omega_{L O}=(4.33 \pm 0.14) \cdot \varepsilon$ and $\Delta \omega_{T O}=(4.16 \pm 0.13) . \varepsilon$ for $\mathrm{GaN}$;

iii) $\Delta \omega_{L O}=(5.51 \pm 0.11) \cdot \varepsilon$ and $\Delta \omega_{T O}=(5.07 \pm 0.09) \cdot \varepsilon$ for InN.

From the above results, we can infer that, for $\mathrm{AlN}$ and $\mathrm{GaN}$, the LO mode has the same behaviour while, for InN, it is more sensitive to the applied strain. For the TO mode, it is interesting to note that, for $\mathrm{GaN}$, it is less sensitive to the applied strain than for AlN. However, the InN TO mode is still the most sensitive one to the applied strain.

The mode Grüneisen parameter can also be obtained from hydrostatic strains as follows,

$$
\gamma=-\frac{d(\ln \omega)}{d(\ln V)}
$$

So, from our results, we found, for the AlN, GaN and InN LO modes, $\gamma=1.03,1.21$ and 1.35 , respectively. And, for the AlN, GaN and InN TO modes, $\gamma=1.39,1.51$ and 1.61 , respectively. Our values compare well with previous theoretical and experimental ones [8]. Also, comparing our results with the experimental values for the $\mathrm{GaN} \gamma_{L O}$ and $\gamma_{T O}$, which are 1.2 and 1.4, respectively [8], the agreement is very good.

We can infer, from our results, that as these $\gamma$ values increase for the increasing cation mass, besides the fact it is 
greater than 1, the III-Nitride optical phonon bands will become flatter, as observed in recent theoretical calculations $[2,7]$.

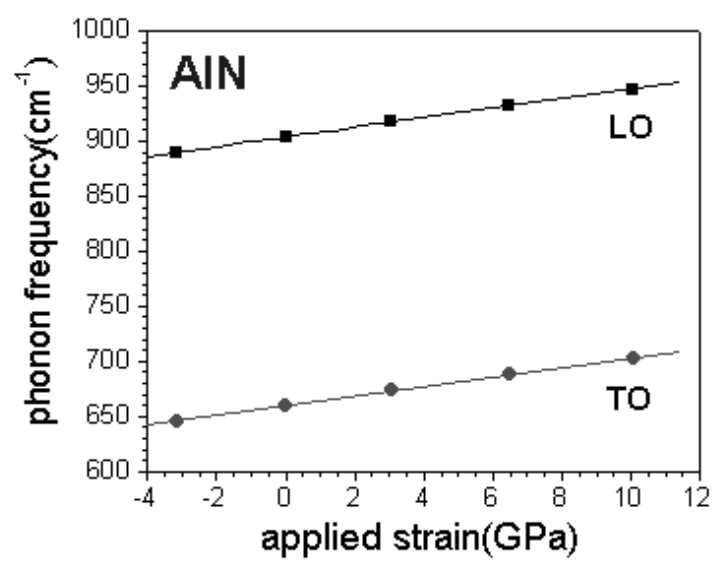

Figure 1. Hydrostatic stress dependence of the frequencies of the zone-center phonons of AlN.

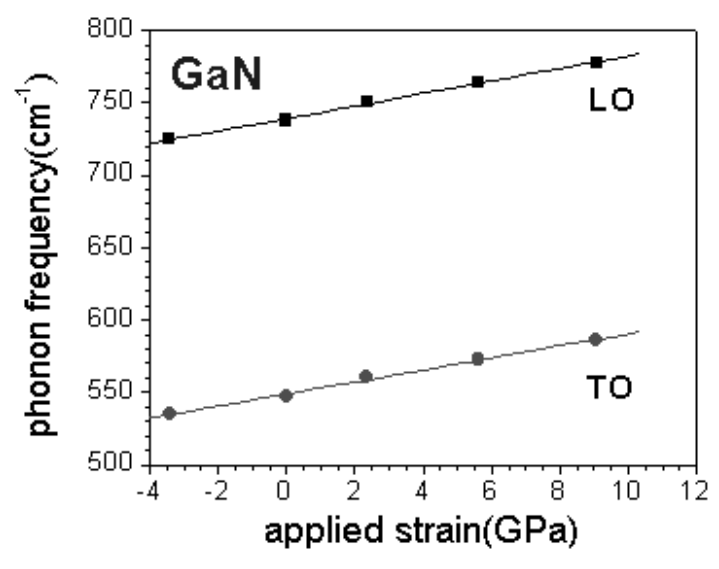

Figure 2. Same as Fig. 1 for zone-center phonons of GaN.

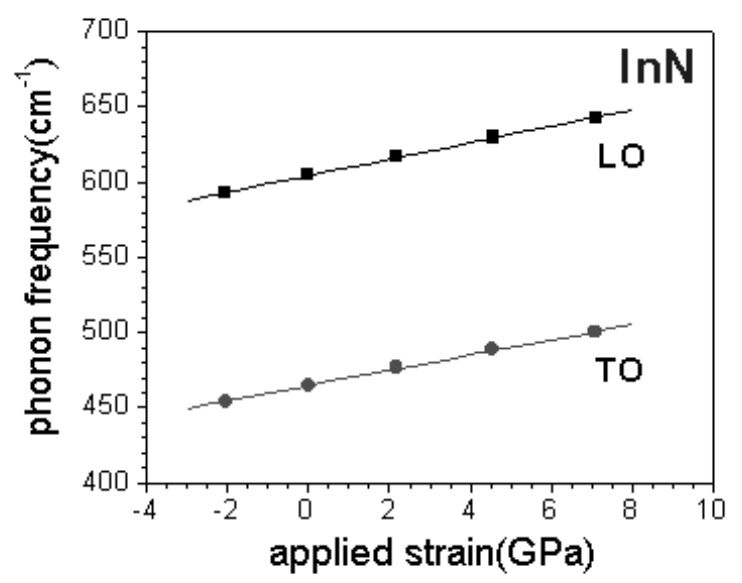

Figure 3. Same as Fig. 1 for zone-center phonons of InN.
In Fig. 4, we show the stress dependence of the cation Born effective charges of AlN, GaN and InN, respectively, under a hydrostatic compressive force. As noted by Wagner et al. [8], the effective charges decrease monotonically with the rising pressure. However, contrary to Ref. 8, the pressure dependence is more pronounced for InN. Moreover, the pressure behaviour of the Born effective charges indicates an electron transfer from the $\mathrm{N}$ atom to the cation in comparison to the pressure-free situation.

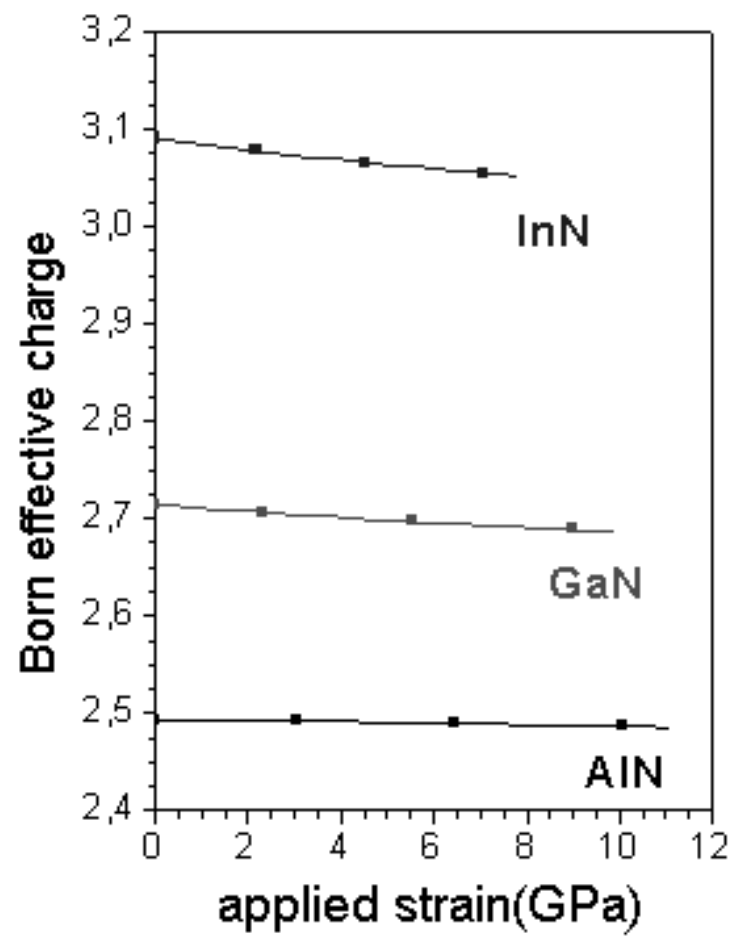

Figure 4. Hydrostatic stress dependence of the cation Born effective charges of III-Nitrides.

\section{Uniaxial strains}

In Fig. 5, we show the stress dependence of the frequencies for the $\operatorname{AlN} \mathrm{TO}(\Gamma)$ mode under a [100] compressive force. We proceed exactly as described in Ref. 9: we first minimized the [010] and [001] lattice parameters when a [100] strain is applied, and then, we evaluated the phonon frequencies at $\Gamma$ point in the final minimized structure. In these specific calculations, we have used a 8-atoms supercell in a cubic symmetry, and a (2 2 2) Monkhorst-Pack grid was used to sample the supercell Brillouin zone.

The obtained stress dependence of the AlN zone-center phonons can be expressed as (in $\mathrm{cm}^{-1} / \mathrm{GPa}$ units):

$$
\begin{aligned}
& \Delta \omega_{L O}=(5.5 \pm 0.9) . \varepsilon \\
& \Delta \omega_{T O 1}=(8.8 \pm 0.8) . \varepsilon, \text { and } \\
& \Delta \omega_{T O 2}=(-3.8 \pm 0.3) . \varepsilon
\end{aligned}
$$

If we compare these results with the hydrostatic strains ones, we can infer that the zone-center vibrational modes of the III-Nitrides are very sensitive to uniaxial strains, since 
we found that the $\mathrm{GaN}$ and $\mathrm{InN}$ phonons are even more sensitive to hydrostatic strains than the AlN ones. Calculations for the [100] strain dependences of the zone-center phonons of $\mathrm{GaN}$ and $\mathrm{InN}$ to check this tendence are currently under way.

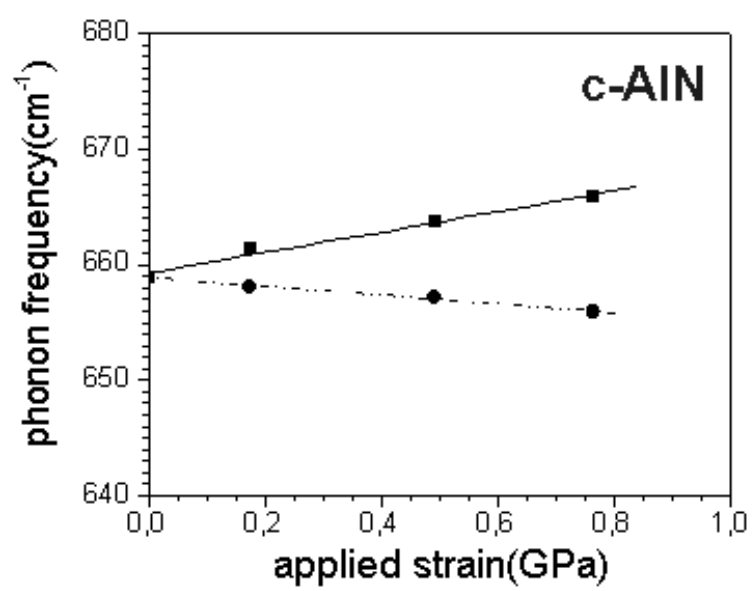

Figure 5. [100] stress dependence of the frequencies of the AlN $\mathrm{TO}(\Gamma)$ mode.

\section{Final Remarks}

We present, here, our preliminary results for the straininduced shifts of the zone center phonon modes and the effective charges on the III-Nitrides. A good agreement with the experiment as well as with other calculations were obtained, whenever a comparison is possible. We hope that our results give guidelines for future experiments on this subject. Further details of our calculations and a careful analysis of the behaviour of the vibration modes under strain will be published soon.

\section{Acknowledgements}

The computer resources were provided by the Centro Nacional de Processamento de Alto Desempenho em Minas Gerais \& Região Centro-Oeste (CENAPAD-MG/CO), Brazil.

\section{References}

[1] S. C. Jain, M. Willander, J. Narayan, and R. van Overstraeten, J. Appl. Phys. 87, 965 (2000).

[2] F. Bechstedt, U. Grossner, and J. Furthmüller, Phys. Rev. B62, 8003 (2000).

[3] X. Gonze, J.-M. Beuken, R. Caracas, F. Detraux, M. Fuchs, G.-M. Rignanese, L. Sindic, M. Verstraete, G. Zerah, F. Jollet, M. Torrent, A. Roy, M. Mikami, Ph. Ghosez, J.-Y. Raty, and D. C. Allan, Comput. Mater. Sci. 25, 478 (2002), and references therein.

[4] C. Stampfl and C. G. Van de Walle, Phys. Rev. B59, 5521(1999).

[5] M. Fuchs and M. Scheffler, Comput. Phys. Commun. 119, 67(1999).

[6] X. Gonze, Phys. Rev. A52, 1096 (1995).

[7] L. S. Pereira, A. M. Santos, J. L. A. Alves, H. W. Leite Alves, and J. R. Leite, Microelect. J. 34, 655 (2003).

[8] J. -M. Wagner, G. Portisch, K. Karch, and F. Bechstedt, Mater. Sci. Eng. B59, 248 (1999), and references therein.

[9] A. T. Lino, N. Meskini, H. W. L. Alves, and K. Kunc, Int. J. Quantum Chem. 45, 43 (1993). 